\title{
Distribuição espacial da tuberculose e sua relação com políticas públicas da atenção básica na mesorregião do Nordeste Paraense-PA/Brasil no período de 2015 a 2019
}

\section{Spatial distribution of tuberculosis and its relationship with public policies of primary care in the mesoregion of the Northeast Paraense-PA-Brazil in the period from 2015 to}

2019

Adriana Veiga da Conceição Silva1 ${ }^{1}$, Luiz Carlos Sousa de Castro², Juan Andrade Guedes $^{3}$, Rafael Aleixo Coelho de Oliveira ${ }^{4}$, Claudia do Socorro Carvalho Miranda ${ }^{5}$, Alan Luz Tembra ${ }^{6}$, Matheus Marinho Rios ${ }^{7}$, Nelson Veiga Gonçalves ${ }^{8}$

\section{RESUMO}

A tuberculose é considerada um grave problema de saúde pública. Este trabalho procurou analisar a distribuição espacial da tuberculose e sua relação com políticas públicas de atenção primária à saúde, na mesorregião do nordeste paraense, no período de 2015 a 2019. Trata-se de um estudo do tipo ecológico, descritivo e transversal que utilizou dados disponíveis em plataformas digitais. Foram confeccionados mapas coropléticos para visualizar a distribuição da doença na região, onde foram utilizadas técnicas de geoprocessamento. As análises de significância estatística foram realizadas com o teste de qui-quadrado: proporções esperadas iguais. O perfil epidemiológico mais acometido foi o de indivíduos do sexo masculino, entre 20 a 59 anos, de cor parda e com escolaridade até o ensino fundamental. Foi observado uma distribuição espacial não homogênea da doença entres os 49 municípios pertencentes a região, estando relacionada com a quantidade de Unidades Básicas de Saúde (UBS). Quanto a cobertura vacinal, foi observado que grande parte dos municípios pertencentes a essa região estão abaixo da meta nacional de imunização pela BCG. Ademais, tais dados proporcionam melhor compressão da distribuição espacial da tuberculose na região sendo importante para a construção dos cenários epidemiológicos e de políticas públicas de saúde, na área de estudo.

Palavras-chave: Tuberculose. Epidemiologia. Geoprocessamento.

\section{ABSTRACT}

Tuberculosis is considered a serious public health problem. Therefore, this work aimed to analyze the spatial distribution of tuberculosis and its relationship with primary health care public policies in the Pará's northeastern mesoregion, on the period from 2015 to 2019. This is an ecological, descriptive and cross-sectional study that used data available on digital platforms. Choropletics maps were made to visualize the distribution of the disease in the region, where geoprocessing techniques were used. The statistical significance analyzes were performed with the chi-square test: expected equal proportions. The most affected epidemiological profile was male individuals, between 20 and 59 years old, brown in color and with schooling up to elementary school. A nonhomogeneous spatial distribution of the disease was observed among the 49 municipalities belonging to the region, which were being related to the number of Basic Health Units (BHU). As for vaccination coverage, it was observed that most of the municipalities belonging to this region were below the national goal of immunization by BCG. Furthermore, such data provide a better comprehension of the spatial distribution of tuberculosis in the region, being important for the construction of epidemiological scenarios and public health policies in this particular area.

Keywords: Tuberculosis. Epidemiology. Geoprocessing
Graduanda em Medicina. Universidade do Estado do Pará (UEPA).

E-mail:

adrianaveigacs@yahoo.com.br

Graduando em Medicina. Universidade do Estado do Pará (UEPA).

Mestre em saúde, ambiente e sociedade na Amazônia (PPGSAS/UFPA). Universidade do Estado do Pará (UEPA).

Mestre em saúde, ambiente e sociedade na Amazônia (PPGSAS/UFPA). Universidade do Estado do Pará (UEPA).

5 Doutora em Biologia Parasitária na Amazônia (PPGBPA/UEPA). Universidade do Estado do Pará (UEPA).

Graduando em Medicina. Universidade do Estado do Pará (UEPA).

Graduando em Medicina. Universidade do Estado do Pará (UEPA).

Docente, Epidemiologista. Universidade Federal Rural da Amazônia, Instituto Ciberespacial, Belém, Pará, Brasil/ Universidade do Estado do Pará, Centro de Ciências Biológicas e da Saúde, Departamento de Saúde Comunitária, Laboratório de Epidemiologia

Geoprocessamento da Amazônia (EPIGEO/UEPA), Belém, Pará, Brasil. 


\section{INTRODUCĀO}

A tuberculose (TB) é uma doença cuja ocorrência remonta às civilizações mais antigas, sendo encontrados registros que apontam para os seus sinais e sintomas, nos relatos de Hipócrates na Grécia antiga, além de escritos chineses e até mesmo em antigos textos hebráicos. ${ }^{1}$

O M. tuberculosis hominis, também denominado de bacilo de Kock (BAAR), é o agente responsável pela maioria dos casos da doença, seu reservatório típico são pessoas com manifestações pulmonares ativas. ${ }^{2} \mathrm{~A}$ transmissão é direta, através da inalação de aerossóis gerados por expectoração ou exposição a secreções contaminadas por pessoas infectadas. ${ }^{2}$ Dessa forma, a doença é considerada um grave problema de saúde pública devido suas complicações, principalmente quando associada a condições sociais e econômicas precárias, causando assim muitas mortes. ${ }^{2}$

A ocorrência da doença nas américas está ligada ao processo de imigração principalmente de europeus e asiáticos. ${ }^{3} \mathrm{Na}$ era moderna, a tuberculose encontrou ambiente favorável para sua transmissão nos grandes centros urbanos. ${ }^{3}$ Este fato está ligado a fatores condicionantes que potencializam a ocorrência da doença, destacando-se as condições socioeconômicas e a dificuldade de acesso a saúde, tais condições expressam a relação da doença com a pobreza, baixa escolaridade, moradias insalubres e adensamento populacional. ${ }^{4}$

O Brasil alcançou a meta dos objetivos de desenvolvimento do milênio para o controle da TB antes de 2015. Dentre os fatores que contribuíram para esse feito esta a implementação da estratégia saúde da família, a partir de 1990. O estabelecimento da atenção básica ou primária à saúde (APS) como porta de entrada do Sistema Único de Saúde (SUS), contribuiu efetivamente para o enfrentamento da doença, através da melhoria do acesso ao diagnóstico e tratamento, todos realizados gratuitamente ${ }^{5}$.

A APS tem como modelo a Política Nacional de Atenção Básica (PNAB) criada em 2006, e atualmente é a principal porta de entrada do SUS, aonde promove ações em saúde que abrange desde a prevenção e promoção a saúde até a reabilitação e manutenção da saúde do indivíduo por meio da equipe multidisciplinar no seu território definido, fortalecendo os princípios e diretrizes do SUS. ${ }^{6}$

Além disso, um dos grandes passos para o controle da doença no país foi a implementação do Programa Nacional de Controle da Tuberculose (PNCT), em 1999, com 
os objetivos de reduzir a morbimortalidade e a transmissão da doença ${ }^{7}$. Para isso, foi utilizado de diversas ferramentas afim de cumprir esses objetivos, tais como, ações educativas, capacitações, ações preventivas em âmbito federal, estadual e municipal, além do aperfeiçoamento da vigilância epidemiológica e melhorias no Sistema de Informação de Agravos de Notificação (SINAN). ${ }^{2}$

Dessa forma, as ações voltadas para o controle da TB no Brasil passaram a ser organizadas por meio dos programas de controle da Tuberculose (PCTs), os quais devem ser guiados por meio de indicadores epidemiológicos e operacionais recomendados pelo Ministério da Saúde (MS). $\cdot^{7-8}$

Entre os indicadores mais utilizados, pode ser citado o coeficiente de incidência, mortalidade, cura e abandono do tratamento, controle de contatos, tratamento diretamente observado (TDO), exames diagnósticos e de acompanhamento, coinfecção TB/HIV e casos de retratamento, os quais possibilitam o monitoramento das ações e resultados obtidos pelo programa, sendo fundamentais na logística do mesmo. ${ }^{7-8}$

O estado do Pará é formado por 144 municípios, que pela Divisão regional do Brasil são subdivididos em mesorregiões e microrregiões geográficas pelo Instituto Brasileiro de Geografia e Estatística (IBGE), sendo essa divisão realizada com a finalidade facilitar o conhecimento geopolítico e agrupa municípios de acordo com as suas similaridades em relação as condições sociais, econômicas e geográficas, com a perspectiva de compreender a organização dos espaços geográficos e facilitar o planejamento e implantação de políticas públicas e privadas nesses espaços. 9-10

Assim, o Pará é dividido em 6 mesorregiões, sendo elas a Metropolitana de Belém, Baixo Amazonas, Nordeste Paraense, Marajó, Sudeste Paraense e Sudoeste Paraense. A mesorregião do Nordeste Paraense tem uma área de 83.316,02 km², que corresponde a 49 municípios do Estado, tem a maior densidade populacional fora da região metropolitana de Belém entre as mesorregiões, com uma população de 1,789,387 habitantes, sendo 873,237 habitantes residentes na zona urbana e 916,150 habitantes na zona rural. 9-10-11

Entender as dinâmicas dos espaços influencia diretamente no comportamento da doença, assim como a existência e eficácia das unidades de saúde, de modo que relacionar essas doenças ao espaço geográfico tem se tornado de grande relevância para o meio científico. ${ }^{11}$ Sendo assim, como alternativa para o entendimento da dinâmica de distribuição dos casos de TB tem sido utilizado, nas últimas décadas, os Sistemas de Informações Geográficas (SIG's). ${ }^{12}$ 
Os SIG's são ferramentas computacionais utilizadas para análise na área da saúde cujo o uso tem histórico recente de uso no Brasil, apresentando inúmeros benefícios no planejamento e análise dos cenários epidemiológicos. Utiliza técnicas computacionais de coleta, tratamento e manipulação de informações sobre doenças infecciosas tais como a TB, além de propiciar o mapeamento de doenças de interesse da saúde coletiva. ${ }^{13-14}$

Ademais, o geoprocessamento e a análise espacial em saúde podem proporcionar um melhor entendimento sobre a distribuição dos casos de TB nessa região do País. Assim, a análise espacial da TB pode gerar informações quantitativas relacionadas a uma determinada localização no espaço geográfico, podendo contribuir para o conhecimento da transmissão e de seus fatores de riscos nos territórios. ${ }^{15-16}$

Dado o exposto, este trabalho procurou analisar a distribuição espacial da tuberculose e sua relação com políticas públicas de atenção básica à saúde, na mesorregião do nordeste paraense, estado do Pará, no período de 2015 a 2019.

\section{MATERIAIS E METODOS}

Neste estudo do tipo ecológico, descritivo e transversal foram utilizados dados epidemiológicos (gênero, raça, faixa etária e escolaridade) do Sistema de Informação de Agravo de Notificação (SINAN). Os dados cartográficos sobre os limites municipais foram obtidos no Censo Demográfico (2010), do Instituto Brasileiro de Geografia e Estatística (IBGE). Os dados referentes aos estabelecimentos de Saúde foram obtidos do Cadastro Nacional dos Estabelecimentos de Saúde (CNES). Já os dados referentes a cobertura vacinal da BCG (Bacilo de Calmette e Guérin) foram extraídos do Sistema de Informações do Programa Nacional de Imunizações (SIPNI).

As localizações dos municípios com casos de Tuberculose e os estabelecimentos de saúde foram georreferenciados laboratorialmente. Na sequência foi realizada a depuração dos dados, para a retirada de inconsistências e incompletudes e posterior indexação dos mesmos em um Banco de Dados Geográficos (BDGEO). Foram realizadas análises descritivas dos dados, com a aplicação do teste estatístico não paramétrico qui-quadrado de proporções esperadas iguais com significância de $0,05 \%$, utilizando o programa Bioestat 5.0.

Na Análise de Dados Espaciais (ADES), a distribuição espacial da doença e das unidades de saúde, bem como da cobertura vacinal foi analisada através de mapas coropléticos, com parâmetros gerados a partir do cálculo de quartis que foram agregados 
em um conjunto de cores, a saber, verde (baixo), amarelo (médio), laranja (alto) e vermelho (muito alto), com o uso do software ArcGis 10.5.1.

Os dados desse estudo compreendem as notificações nos municípios do Nordeste Paraense no período de 2015 a 2019, sendo expluídos casos notificados em outros municípios ou em anos anteriores a 2015 ou posterior a 2019.

O estudo contou com dados de domínio público, portanto não foram coletados, manipulados ou utilizados materiais biológicos de espécie alguma, portanto não foi necessário o parecer de aprovação pelo Comitê de Ética em Pesquisa.

\section{RESULTADOS E DISCUSSÃO}

Quanto ao perfil epidemiológico, a Tabela 1 mostra a maior ocorrência da TB no gênero masculino e na faixa etária de 20-59 anos sugere um maior grau de exposição destes indivíduos aos fatores de risco da doença, sobretudo, devido a inserção em atividades laborais em locais cuja veiculação da doença está presente. Essa tendência da doença também pode estar associada às características comportamentais dos homens relacionadas ao tabagismo, alcoolismo, uso de drogas ilícitas. ${ }^{17}$

A maior ocorrência da doença em pessoas pardas como mostra a Tabela 1, pode ser justificada pelo maior quantitativo populacional dessa etnia na Amazônia. Nos municípios do Pará, este fato está relacionado ao processo histórico de miscigenação de indígenas, europeus e africanos, motivo pelo qual $81,77 \%$ da população desse estado se autodeclara parda. Resultados semelhantes foram encontradas em outros estudos, como os de Costa ${ }^{18}$ e Aragão ${ }^{19}$.

A maior parte dos casos notificados apresentou baixa escolaridade, indicando a relação entre esses indivíduos e as condições de vulnerabilidade social das populações que habitam nesses municípios, o que pode favorecer a transmissão da doença. Essas características reforçam a tuberculose como marcador de iniquidades sociais em saúde, de modo que a desigualdade social interfere diretamente no processo de adoecimento, principalmente no que tange a cadeia de transmissão, com base nos determinantes sociais em saúde. ${ }^{20}$ 
DOI: 10.18606/2318-1419/amazonia.sci.health.v9n2p107-120 Revista Amazônia Science \& Health 2021, Vol. 9, № 2 .
SILVA, AVC. CASTRO, LCS. GUEDES, JÁ. OLIVEIRA, RAC. MIRANDA, CSC. TEMBRA, AL. RIOS, MM. GONÇALVES, NV.

Distribuição espacial da tuberculose e sua relação com políticas públicas da atenção básica na mesorregião do Nordeste Paraense-PA/Brasil no período de 2015 a 2019.

Tabela 1.perfil epidemiológico dos casos de tuberculose no nordeste paraense, 2015-2019.

\begin{tabular}{clccc}
\hline Variáveis & & $\mathbf{n = 3 7 3 5}$ & $\%$ & p-valor \\
\hline \multirow{2}{*}{ Gênero } & Masculino & $\mathbf{2 4 2 9}$ & $\mathbf{6 5 , 0 3}$ & $<0.0001$ \\
& Feminino & 1306 & 34,97 & \\
& Ign/Branco & & & \\
\multirow{5}{*}{ Raça/Cor } & 84 & 2,25 & \\
& Branca & 302 & 8,08 & \\
& Preta & 267 & 7,15 & $<0.0001$ \\
& Amarela & 19 & 0,51 & \\
& Parda & $\mathbf{3 0 5 4}$ & $\mathbf{8 1 , 7 7}$ & \\
& Indígena & 9 & 0,24 & \\
& & & & \\
& Ign/Branco & 1 & 0,03 & \\
& $0-9$ anos & 88 & 2,36 & \\
& $10-19$ anos & 331 & 8,86 & $<0.0001$ \\
& 20-59 anos & $\mathbf{2 6 7 7}$ & $\mathbf{7 1 , 6 8}$ & \\
& $\geq 60$ anos & 638 & 17,07 & \\
& & & & \\
& Ign/Branco & 808 & 21,63 & \\
& Analfabeto & 245 & 6,56 & \\
Escolaridade & Ensino fundamental & $\mathbf{1 9 0 7}$ & $\mathbf{5 1 , 0 6}$ & $<0.0001$ \\
& Ensino médio & 595 & 15,93 & \\
& Educação superior & 108 & 2,89 & \\
& Não se aplica & 72 & 1,93 & \\
\hline
\end{tabular}

Fonte: Protocolo de pesquisa/EpiGeo/CCBS/UEPA

Em conjunto, os achados quanto ao perfil dessa população sugerem tratar-se de indivíduos com baixo nível sócio econômico, com grande exposição a pessoas com tuberculose ativa e vivendo em condições que favorecem a propagação da doença. Essas características reforçam a tuberculose como marcador de iniquidades sociais em saúde, de modo que a desigualdade social interfere diretamente no processo de adoecimento, principalmente no que tange a cadeia de transmissão, com base nos determinantes sociais em saúde, principalmente no que se refere a baixas condições de vida. ${ }^{4}$

Os casos de tuberculose no período de estudo ocorreram em todos os 49 municípios da região do nordeste paraense. $\mathrm{Na}$ análise da distribuição espacial dos casos foi evidenciada uma distribuição não-homogênea dos mesmos nos municípios da mesorregião estudada, como mostra a Figura 1. Sendo que as maiores quantitativos de casos ocorreram nos municípios de Vigia, Curuçá, Igarapé Açú, Bragança, Capanema, Salinópolis, São Miguel do Guamá, Tomé-açú, Abaetetuba, Cametá, Igarapé-miri e Tailândia. Assim, a distribuição da doença ocorreu em 5 microrregiões região nordeste paraense. 
DOI: 10.18606/2318-1419/amazonia.sci.health.v9n2p107-120 Revista Amazônia Science \& Health 2021, Vol. 9, № 2 .
SILVA, AVC. CASTRO, LCS. GUEDES, JÁ. OLIVEIRA, RAC. MIRANDA, CSC. TEMBRA, AL. RIOS, MM. GONÇALVES, NV.

Distribuição espacial da tuberculose e sua relação com políticas públicas da atenção básica na mesorregião do Nordeste Paraense-PA/Brasil no período de 2015 a 2019.

Nesta análise, também foi possível evidenciar a distribuição das Unidades Básicas de Saúde (UBS) na região de estudo. Sendo que os municípios apresentaram diferenças com relação a presença de estabelecimentos de saúde, sendo observada a inexistência destes serviços nos municípios de Garrafão do Norte e Ourém que apresentaram baixo, Mãe do Rio que apresentou médio e Tracuateua que apresentou alto números de casos de Tuberculose notificadas no período de estudo (FIGURA 1).

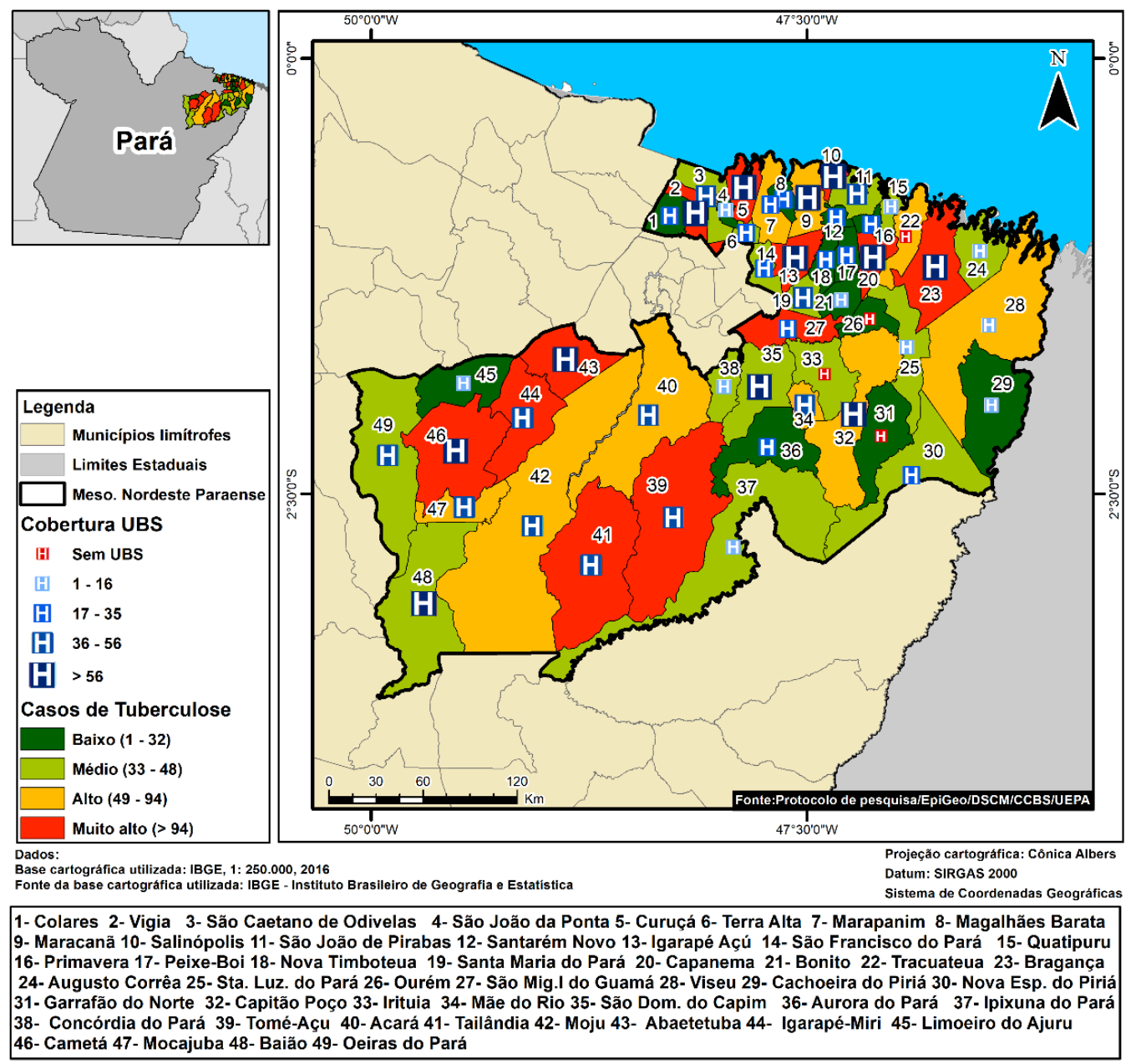

Figura 01 - Distribuição de casos de tuberculose e das Unidades Básicas de Saúde nos municípios do Nordeste Paraense, no período de 2015 a 2019.

Fonte: Protocolo de pesquisa/EpiGeo/CCBS/UEPA, 2021. 
O fato dos municípios de Garrafão do Norte e Ourém terem apresentado baixo número de casos e Mãe do Rio ter apresentado médio pode estar relacionado ao fato de municípios aonde há a ausência ou baixa cobertura de UBS apresentar subnotificações de seus agravos. Esta situação se constitui de problema para a Vigilância Epidemiológica da doença. Pois, no âmbito das ações realizadas pelas UBS e de suas equipes é realizado o acompanhamento e a busca ativa de casos de doenças de notificação compulsória. Sendo que as ações de vigilância epidemiológicas dependem dessas informações para produzir ações em saúde. ${ }^{21-22}$

Neste cenário, uma vez que identificado o agravo de notificação compulsória, a sua vigilância deve ser realizada pelo município onde está sendo notificado o caso, mesmo que este não seja o município que o paciente reside, sendo assim, quando há a procura por atendimento em outras localidades, estes devem alimentar o SINAN. ${ }^{22}$

É importante entender que a responsabilidades da notificação é de todos os profissionais de saúde ou qualquer cidadão, e a coleta de dados se dá em todos os níveis de atenção à saúde, podendo o mesmo ocorrer nas UBS e em diferentes locais como nas Unidades Saúde da Família, centros de saúde, Hospitais, centros de referência, institutos, laboratórios, centros de reabilitação e outros serviços de assistência médica do SUS ou particular. ${ }^{22-23}$

Analisar a ocorrência de agravos as condições de saúde do indivíduo no local aonde ele vive, por meio das UBS, ESF dos municípios possibilita identificar diferentes fatores sociais, sanitários e epidemiológicos relacionados as suas necessidades em saúde nas ações contra a tuberculose. Mas para este fim, são necessários locais de atendimento e profissionais de saúde para atender essa população e ser um meio de compartilhamento de informações para os sistemas de notificação e também de participação da população, aumentando os seus acessos e informações além de favorecer o seu monitoramento. .23-24 $^{24}$

Foi evidenciado um aumento do número de casos de TB e do quantitativo de UBS nos municípios do nordeste paraense ao longo do período de 2015 a 2019 como mostra a Figura 2. Esse fato pode estar relacionado ao aumento do fluxo migratório ocorrido para a região ao longo dos anos e a uma maior busca ativa de casos pelas equipes multiprofissionais de Saúde da Atenção Básica. Esse resultado contrapõe os estudos de Neves $^{25}$, que mostrou uma tendência a redução do número de casos nos municípios paraenses, com exceção da região metropolitana no período de 2005 a 2014. 


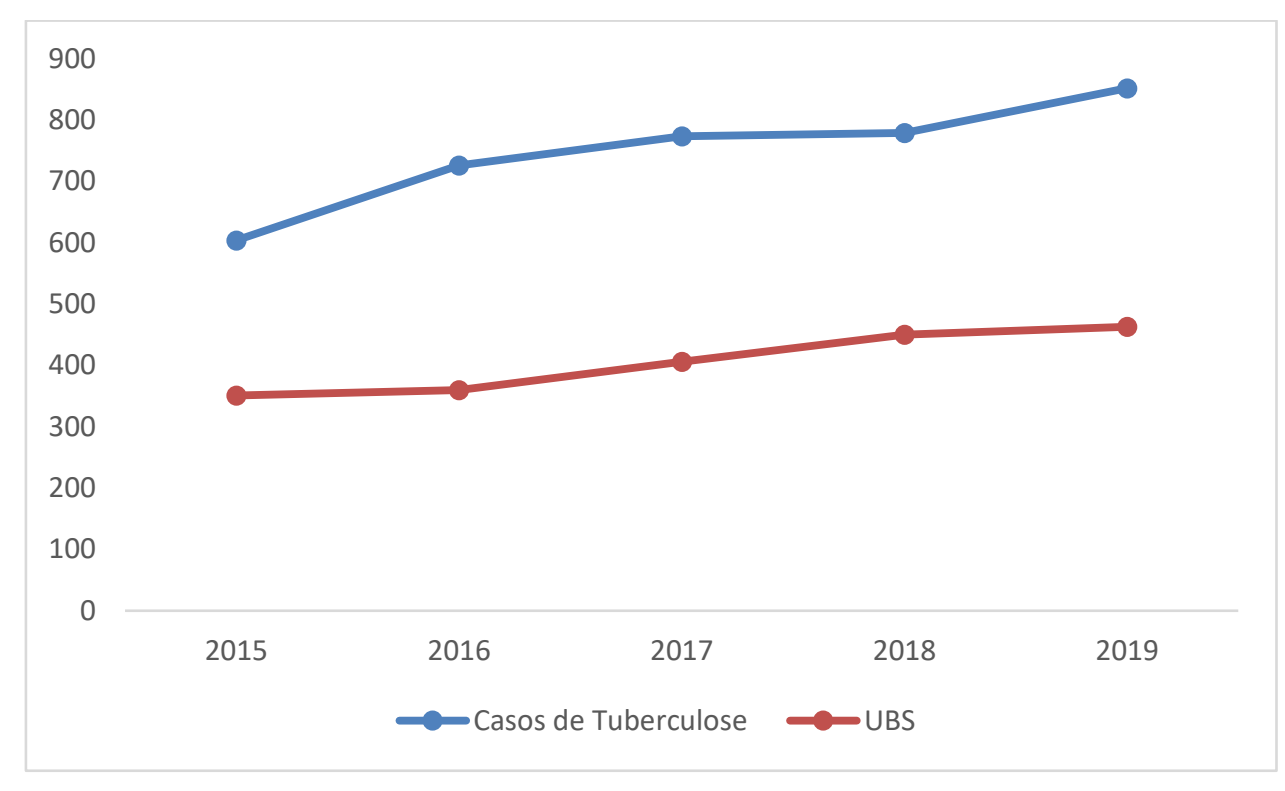

Figura 02 - Número de Casos de Tuberculose e de Unidades Básicas de Saúde (UBS) nos municípios do Nordeste Paraense, nos anos de 2015 a 2019.

Fonte: Protocolo de pesquisa, 2021.

O aumento da quantidade de UBS é uma importante ferramenta de análise epidemiológica, pois funcionam como um importante meio de análise da vigilância em saúde, através da alimentação dos sistemas de informação e de notificação da doença. Com a análise do perfil epidemiológico é possível alocar recursos e traçar estratégias como a disponibilidade de vacinas, medicamentos, ações de educação em saúde que atendam a sua população e a contenção da transmissão pelas correntes migratórias e fluxo de transmissão. ${ }^{26}$

Outra importante política pública da atenção básica relacionada as melhores condições de vida é a vacinação com a BCG, pois foi evidenciado muito alta cobertura vacinal nos municípios de São Caetano de Odivelas, Quatipuru, Peixe- boi, Bonito, Bragança, Santa Luzia do Pará, Ourém, Nova Esperança do Piriá, Capitão poço, Concórdia do Pará, Tailândia e Cametá. Já em Curuçá, Terra alta, Marapanim, Igarapé Açú, Santa Maria do Pará, Cachoeira do Piriá, Irituia, São Domingos do Capim, Acará, Mojú, Abaetetuba, Limoeiro do Ajurú e Mocajuba foi identificada baixa cobertura vacinal conforme mostra a Figura 3.

A baixa cobertura vacinal, aliada a ausência ou insuficiência da quantidade de UBS leva ao maior risco do adoecimento pelo bacilo. A maioria dos municípios no Nordeste Paraense estão com a imunização abaixo da meta Nacional de imunização pela BCG. 
DOI: 10.18606/2318-1419/amazonia.sci.health.v9n2p107-120 Revista Amazônia Science \& Health 2021, Vol. 9, № 2 .
SILVA, AVC. CASTRO, LCS. GUEDES, JÁ. OLIVEIRA, RAC. MIRANDA, CSC. TEMBRA, AL. RIOS, MM. GONÇALVES, NV.

Distribuição espacial da tuberculose e sua relação com políticas públicas da atenção básica na mesorregião do Nordeste Paraense-PA/Brasil no período de 2015 a 2019

Cobertura inferior ao dos estudos de Oliveira ${ }^{27}$, que encontrou uma cobertura vacinal da BCG no Norte do Brasil de $80 \%$

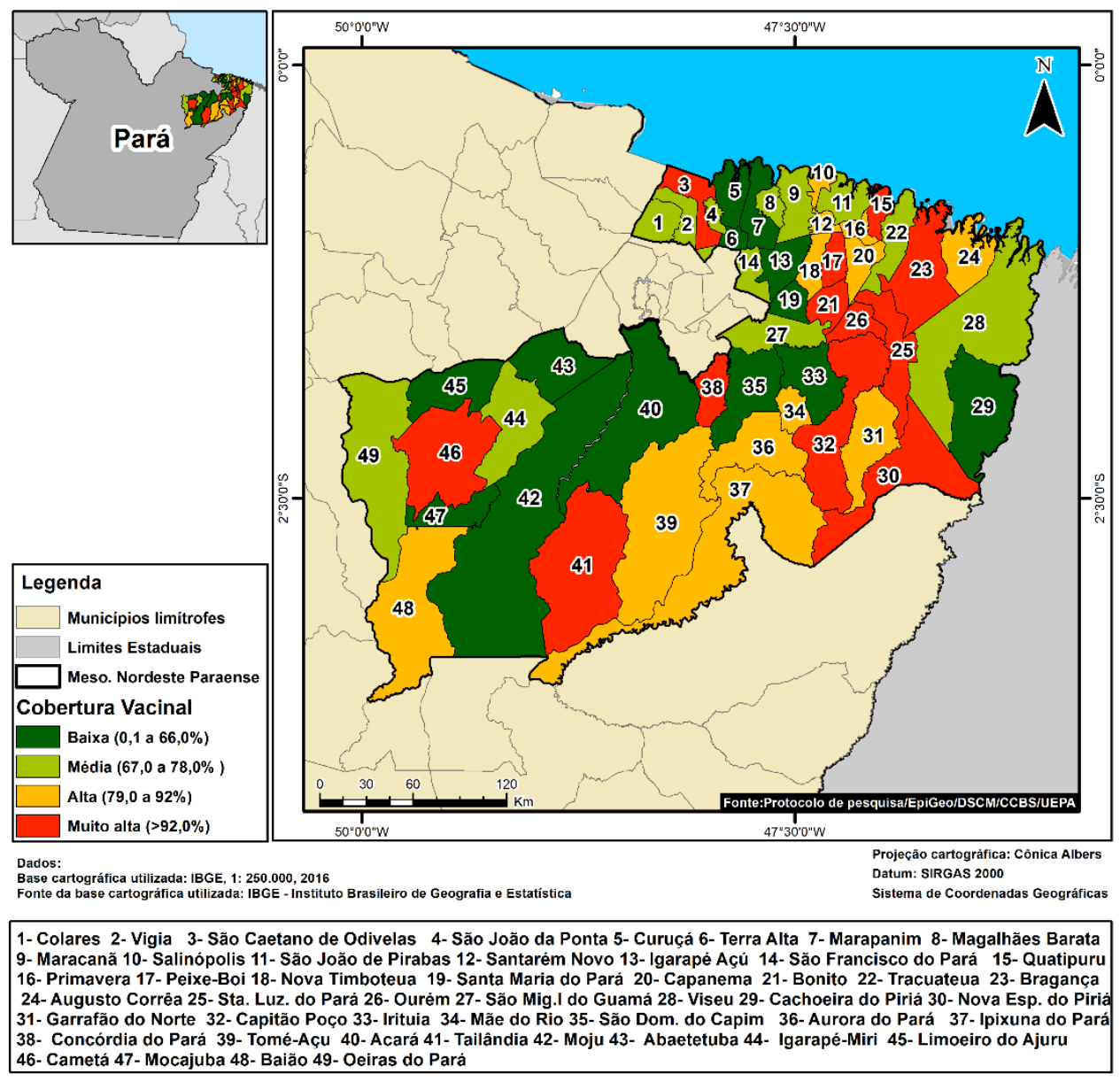

Figura 03 - Distribuição da cobertura em porcentagem (\%) da Imunização por BCG nos municípios do Nordeste Paraense, no período de 2015 a 2019.

Fonte: Protocolo de pesquisa/EpiGeo/CCBS/UEPA, 2021.

A baixa imunização, aliada a ausência ou baixo quantitativo de UBS nos municípios, levam ao maior risco do adoecimento e também das complicações pela Tuberculose. Nesse contexto, o Programa Nacional de Controle da Tuberculose (PNCT), tem como objetivos específicos garantir a cobertura adequada de BCG, prevenir o adoecimento nos infectados por meio da quimioprofilaxia, além de reforçar a importância de alimentar o sistema de informação e de avaliações epidemiológicas. ${ }^{28}$

Avaliar os programas de imunização através do monitoramento da cobertura vacinal é essencial para estimar a efetividade do sistema de saúde. ${ }^{29} \mathrm{Na}$ população estudada, foi 
observado casos de Tuberculose não apenas nos indivíduos adultos, mas em todas a faixa etária, fato este que possivelmente pode estar relacionado a uma baixa cobertura vacinal, baixas condições de vida e de ações e estrutura das Unidades Básicas de Saúde.

Nesse contexto, o conhecimento da cobertura vacinal pode representar um termômetro, indicando a maior ou menor presença de políticas voltadas ao controle da tuberculose, de modo que identificar as áreas com menos cobertura vacinal contribui para tomada de decisões e planejamento de ações direcionadas a populações específicas. ${ }^{284}$

\section{CONSIDERAÇÓES FINAIS}

Neste estudo, foi analisado a distribuição espacial da Tuberculose e a sua relação com as políticas públicas de saúde nos municípios da mesorregião do Nordeste Paraense, no período e 2015 a 2019. Os resultados evidenciaram que a ocorrência da doença nesta região é um grande problema de saúde pública. O perfil epidemiológico identificado com maior prevalência foi o de indivíduos do sexo masculino, entre 20 a 59 anos, de cor parda e com escolaridade até o ensino fundamental.

Foi observado uma distribuição espacial não homogênea da doença entres os 49 municípios pertencentes no Nordeste Paraense, que está diretamente relacionada com a quantidade de Unidades Básicas de Saúde (UBS), onde a maior presença das UBS implica também em um maior rastreio e notificação de casos. Quanto a cobertura vacinal, foi observado que grande parte dos municípios pertencentes a essa região estão abaixo da meta nacional de imunização pela BCG. Tais achados tornam-se preocupante haja vista que a menor cobertura vacinal leva ao maior risco de adoecimento.

As ferramentas computacionais utilizadas na análise espacial dos dados foram satisfatórias para a construção dos cenários epidemiológicos da Tuberculose e das políticas públicas de saúde, na área de estudo. Desta forma, estas apresentam grande potencial para prover os gestores em saúde com informações voltadas para a vigilância contínua e sistemática do agravo estudado.

Considerando as relações entre as variáveis analisadas neste estudo, o desenvolvimento de outros estudos em escala local é necessário para mitigar a doença nos municípios estudados, apesar das dificuldades estruturais como grandes distâncias e acessos. 
Levando em consideração os resultados obtidos ao longo do desenvolvimento deste trabalho ressaltamos a necessidade da intensificação de medidas e ações de vigilância epidemiológica nas áreas onde foram observadas a produção da doença.

\section{REFERÉNCIAS}

1. KozakeilchH GV, Silva RM. Tuberculose: revisão de literatura. Arquivos Catarinenses de Medicina, Santa Catarina. 2015; 44(4): 34-37.

2. Brasil. Ministério da Saúde. Secretaria de Vigilância em Saúde. Departamento de Vigilância das Doenças Transmissíveis. Manual de Recomendação para o Controle da Tuberculose no Brasil 2. ed. Brasília: Ministério da Saúde, 2019, 364 p.

3. Pelissari DM. Impacto do encarceramento na incidência da tuberculose. São Paulo. Tese Doutorado [Curso de Pós Graduação em Epidemiologia] - Universidade de São Paulo; 2019.

4. André SR, Nogueira LMV, Rodrigues ILA, Cunha TN, Palha PF, Santos CB. Tuberculosis associated with the living conditions in an endemic municipality in the North of Brazi. Rev. Latino-Am. Enfermagem. 2020;28:e3343.

5. Maciel ELN, Sales CMM, Bertolde Al, Santos BR. Can Brazil achieve the new World Health Organization global targets for tuberculosis control?. Epidemiol. Serv. Saúde. 2018; 27(2):e0200007.

6. Macinko J, Mendonça CS. Estratégia Saúde da Família, um forte modelo de Atenção Primária à Saúde que traz resultados. Saúde Debate, Rio De Janeiro, set 2018. V. 42, n 1: p. 18-37.

7. Ferreira MRL, Bonfim RO, Orfão NH. Desempenho dos Programas de Controle da Tuberculose: Revisão Integrativa da Literatura. Rev. Contexto \& Saúde. 2020; 41(20): 134143.

8. BrasiL. Ministério da Saúde. Secretaria de Vigilância em Saúde. Departamento de Vigilância das Doenças Transmissíveis. Brasil livre da Tuberculose: Plano Nacional pelo Fim da Tuberculose como Problema de Saúde Pública. Brasília: Ministério da Saúde, 2017. $52 \mathrm{p}$.

9. Cordeiro IMCC, Rangel-Vasconcelos LGT, Schwartz G, Oliveira FA. Nordeste Paraense: Panorama geral e uso Sustentável das florestas Secundárias - Belém: EDUFRA, 2017. 323p.: il.

10. Instituto Brasileiro de Geografia e Estatística - IBGE. Projeto Levantamento e Classificação do Uso da Terra Uso da Terra no Estado do Pará Relatorio Tecnico, Rio de Janeiro, 2013. 
DOI: 10.18606/2318-1419/amazonia.sci.health.v9n2p107-120 Revista Amazônia Science \& Health 2021, Vol. 9, № 2 .
SILVA, AVC. CASTRO, LCS. GUEDES, JÁ. OLIVEIRA, RAC. MIRANDA, CSC. TEMBRA, AL. RIOS, MM. GONÇALVES, NV.

Distribuição espacial da tuberculose e sua relação com políticas públicas da atenção básica na mesorregião do Nordeste Paraense-PA/Brasil no período de 2015 a 2019.

11. Censo Demográfico 2010. Características gerais da população, religião e pessoas com deficiência. Rio de Janeiro: IBGE, 2010. Acompanha 1 CD-ROM. Disponível em: https://biblioteca.ibge.gov.br/visualizacao/periodicos/94/cd 2010 religiao deficiencia.pdf.

Acesso em: abril. 2021.

12. Leal BN, Mesquita CR, Nogueira LM, Rodrigues ILA, Oliveira LF, Caldas, RJC. Análise espacial em tuberculose e a rede de atenção primária em saúde. Rev. Bras. Enferm. 2019; 72(5): 1262-1267.

13. Gonçalves NV, Araujo EN, Sousa Júnior AD, Pereira WM, Miranda CD, Campos OS, et al. Distribuição espaço-temporal da leptospirose e fatores de risco em Belém, Pará, Brasil. Cien Saude Colet. 2016;21(12):3947-55.

14. Brasil. Ministério da Saúde. Fundação Oswaldo Cruz. Abordagens espaciais na saúde pública / SANTOS S. M. \& BARCELLOS, C., Organizadores. - Brasília : Ministério da Saúde, 2006. 136 p.: il. Volume 1.

15. Bonfim C, Medeiros Z. Epidemiologia e geografia: dos primórdios ao geoprocessamento. Espac Saude. p. 53-62, 2008v. 10, n. 1.

16. Sousa AIA, Pinto Júnior VL. Análise espacial e temporal dos casos de aids no Brasil em 1996-2011: áreas de risco aumentado ao longo do tempo. Epidemiol e Serv de Saúde. 2016v. 25, p. 467-476.

17. Silva DR, Muñoz-Torrico M, Duarte R, Galvão T, Bonini EH, Arbex FF, Arbex MA, Augusto VM, Rabahi MF, Mello FCQ. Fatores de risco para tuberculose: diabetes, tabagismo, álcool e uso de outras drogas. J Bras Pneumol. 2018;44(2):145-152.

18. Costa RJF, Miranda CSC, Noguchi SKT, Oliveira RAC, Santos BO, Pinto DS. Distribuição Espaço-Temporal da Tuberculose no Município de Ananindeua, Pará, Brasil. Rev. Insp. Movim. \&. Saúde. 2021; 21(1): 1-16.

19. Aragão FBA, Arcêncio RA, Carneiro TG, Cândido, FCA, Cunha JHS, Reis AD, Fiorati RC. (2020). Análise epidemiológica da tuberculose em São Luís-MA. Medicina (Ribeirão Preto), 53(3), 252-259.

20 Oliveira MSR, Sousa LC, Baldoino LS, Alvarenga AA, Silva MNP, Elias ACG, et al. Perfil epidemiológico dos casos de tuberculose no estado do Maranhão nos anos de 2012 a 2016. Rev. Prev. Infec e Saúde. 2018; 4: 6896.

21. Texeira MG, Penna GO, Risi JB, Penna ML, Alvim MF, Moraes JC, Luna E. Seleção das doenças de notificação compulsória: critérios e recomendações para as três esferas de governo. Inf. Epidemiol. Sus. 1998; 7(1) v.7 n.1: 7-28.

22. Brasil. Ministério da Saúde. Secretaria de Vigilância em Saúde. Departamento de Vigilância Epidemiológica. Guia de vigilância epidemiológica 7. ed. Brasília: MS, 2009. 816 p.

23. Brasil. Ministério da Saúde Secretaria de Vigilância em Saúde. Departamento de Vigilância Epidemiológica. Manual de recomendações para o controle da tuberculose no Brasil. Brasília: Ministério da Saúde ; 2011. 284 p. 
24. Bertolozzi M R, Faria LS. A vigilância na Atenção Básica à Saúde: perspectivas para o alcance da Vigilância à Saúde*. Revista Escola de Enfermagem USP. 2010.

25. Neves DCO, Loureiro LO, Paica NP, Ohnishi MDO, Ribeiro CDT. Análise do Programa de Controle da Tuberculose no estado do Pará, Brasil, de 2005 a 2014. Rev. PanAmazônica de Saúde. 2018; 8(4): :47-56.

26. Ferreira MC, Zuben APBV. Vigilância em saúde nos municípios: caderno de textos . 1. ed. -- Campinas, SP : IPADS, 2020.

27. Oliveira GS, Bitencourt EL, Amaral PFF, Vaz GP, Reis Júnior PM. Cobertura vacinal: uma análise comparativa entre os Estados da região Norte do Brasil. Revista de Patologia do Tocantins. 2020; Vol.7. No.1, junho: 14-17.

28. Brasil. Ministério da Saúde. Secretaria de Vigilância em Saúde. Departamento de Vigilância Epidemiológica. Manual de recomendações para o controle da tuberculose no Brasil / Ministério da Saúde, Secretaria de Vigilância em Saúde, Departamento de Vigilância Epidemiológica. - Brasília : Ministério da Saúde, 2011. 284 p. : il.

29. Queiroz LLC, Monteiro SG, Mochel EG, Veras MASM, Sousa FGM, Bezerra MLM, et al. Cobertura vacinal do esquema básico para o primeiro ano de vida nas capitais do Nordeste brasileiro. Caderno de Saúde Pública. 2013; 29(2): :294-302. 